\title{
Omega-3 in The Treatment of Mood and Sleep Disorders Induced by Hormone Therapy in Women with Breast Cancer: A Randomized, Double-Blinded, Placebo-Controlled Clinical Trial
}

Behzad Nourosi

Isfahan University of Medical Sciences

Erfan Naghsh

Ryerson University

Sahar Esmaeil zadeh

Isfahan University of Medical Sciences

Valiollah Mehrzad

Isfahan University of Medical Sciences

Ali Darakhshandeh

Isfahan University of Medical Sciences

Azadeh Moghaddas ( $\nabla$ azadeh_moghaddas@yahoo.com )

Isfahan University of Medical Sciences https://orcid.org/0000-0003-0412-6172

\section{Research Article}

Keywords: Omega 3, Mood disorder, Sleep quality, Breast cancer

Posted Date: November 12th, 2021

DOI: https://doi.org/10.21203/rs.3.rs-1055943/v1

License: (c) (i) This work is licensed under a Creative Commons Attribution 4.0 International License.

Read Full License 


\section{Abstract \\ Purpose}

This study was performed to investigate the effect of omega-3 on the treatment of mood and sleep disorder induced by menopausal complications due to hormone therapy in patients with breast cancer.

\section{Methods}

A placebo, double-blind and controlled trial was designed in oncology-hematology outpatient's clinic of Omid Hospital, Isfahan, Iran. First, sixty patients were screened by hospital anxiety and depression scale (HADS) for any baseline mood disorders and then divided into either intervention group who had received 2 grams' omega-3 daily for 4 weeks or identical placebo. Then, the patients were considered to assess by center for epidemiological studies-depression scale (CES-D), profile of mood states (POMS), and Pittsburgh sleep quality index (PSQI) questionnaires at the baseline and after 4-week follow-up.

\section{Results}

Findings showed that the mean scores of CES-D $(P=0.002)$, POMS $(P=0.03)$, and PSQI $(P=0.04)$ were significantly lower in the intervention group than the control group. In the intervention group, mean scores of CES-D ( $P$ <0.001), POMS ( $P<0.001)$, and PSQI $(P=0.003)$ were significantly lower in post-intervention than pre-intervention. Mean changes in scores of CES-D $(P=0.01)$, POMS $(P=0.001)$, and PSQI $(P=$ 0.02 ) were significantly higher in the intervention group than the control group.

\section{Conclusion}

Our findings revealed that omega-3 supplementation have the potential to reduce mood disorders as well as to improve sleep quality in terms of subjective sleep quality, delay in falling asleep, sleep delay, and sleep disturbance in patients with breast cancer.

\section{Introduction}

Breast cancer is known as the most common life-threatening cancer in women. It is also the most common cause of cancer-related deaths in women in developing countries and is the most prevalent type of cancer after lung cancer in developed countries [1]. The overall increase in incidence of breast cancer has increased rapidly in the last decades due to the widespread use of mammography screening technique. Various methods, such as chemotherapy, surgery, radiotherapy, and hormone therapy are used in the treatment of breast cancer patients [2]. At present, the use of estrogen receptor inhibitors is considered as the main treatment for breast cancer with positive hormone receptors and are used along with other treatments. Tamoxifen as a selective estrogen receptor modulator and aromatase inhibitors 
(Als) are utilized as a standard drug in the treatment of hormone receptor-expressing breast cancer patients. Long term use of hormone therapy poses serious side effects such as hot flashes, night sweats, mood and sleep disturbances.

The effects of menopausal symptoms on women's quality of life can worsen perception of general health, energy, social functioning, mental health, depression, and sexual satisfaction.

At present, various drugs, such as selective serotonin reuptake inhibitors, venlafaxine, sertraline, paroxetine, and some anticonvulsant drugs, such as gabapentin and pregabalin were addressed to use for the side effects induced by hormone therapy in breast cancer patients [3, 4].

Omega-3 supplements contain polyunsaturated fatty acids (PUFAs), which include longer-chain fatty acids, such as eicosapentaenoic acid (EPA) and docosahexaenoic acid (DHA), as well as alpha-linolenic acid (ALA) [5]. These fatty acids are the main components of phospholipid membranes that are effective in organizing or separating molecules and influence cell signaling and also play a role in the production of bioactive fat mediators in relieving inflammation [6]. Omega-3 fatty acids are considered to be the most widely suggested supplements in the treatment of cardiovascular disease, breast cancer, rheumatoid arthritis, depression, and other cognitive disorders [7]. Clinical data have shown that the use of omega-3 fatty acids enhance the anti-tumor effects of tamoxifen by inhibiting several proliferative and anti-apoptotic pathways and many of these pathways interact with estrogen receptor signaling [8]. Supplementation of omega-3 fatty acids resulted in lower toxic doses of tamoxifen [5].

Positive effects and safe administration of omega-3 supplementation in the control of menopausal symptoms and mood changes in non-cancerous postmenopausal women have been addressed in several studies [9-12]. However, there is still no enough evidences on positive effects of omega-3 supplementation on breast cancer patients' undergoing treatment with anti-hormone therapy specially in term of mood and sleep disorders. Furthermore, this study was conducted to investigate the effect of omega-3 supplementation on the treatment of mood changes and sleep disorders caused by menopausal complications due to hormone therapy in patients suffering from breast cancer.

\section{Methods}

\section{Participants and study design:}

This study was designed in randomized, double blinded, placebo-controlled clinical trial. The patients' recruitment was carried out from March 2020 to March 2021 among outpatients in hematology-oncology clinic of Omid hospital, Isfahan, Iran. Omid hospital is a 200-beds, referral, and university-affiliated hospital specialized for the treatment of hematology-oncology patients. The protocol of study has been approved in Isfahan University of Medical Sciences ethic committee (ID number: IR.MUI.RESEARCH.REC.1399.362) and also trial was submitted in Iranian clinical trial registry center (IRCT20180722040556N6). 
We included adult patients (18 years and older) suffering from hormone-positive breast cancer of any degree (I to III) who had completed all course of chemotherapy and radiotherapy treatment while they were under the treatment by hormone therapy.

Hormone therapy was included by administration of selective estrogen receptor modulators (SERM) family drugs (including tamoxifen) or aromatase inhibitors (including steroidal and non-steroidal agents). Before starting recruitment, all patients who had signed the consent form firstly were screened by hospital anxiety and depression scale (HADS), translated and validated by Montazeri et al [13]. The HADS is a fourteen-item scale widely used for measuring psychological morbidity in cancer patients. Scoring for each item ranges from zero to three.

If the patients' score was more than 11 in the early evaluation, the patient was asked to consult with a psychiatrist, if not, he or she were invited to participate in our study if they were complaining of mood or sleep changes during one month ago.

The exclusion criteria were included, patients with a history of any other malignancies, patients receiving anticonvulsant medications due to their active seizures, patients who had any known psychiatric problems (depression, bipolar, psychosis and etc.) or those who were receiving medications that affected the central nervous system, such as antipsychotics, hypnotic or sedative (such as benzodiazepines, nonbenzodiazepines agents or any drugs with antihistamine properties) or antidepressants and anti-anxiety. We also excluded patients who were receiving drugs such as propranolol (due to the relative effect on the central nervous system) and warfarin (due to the high risk of interactions), any supplements such as soy supplements or vitamin E to reduce menopausal symptoms. Patients with any degree of liver and kidney failure and autoimmune diseases and those who had a history of smoking, chronic alcohol consumption were also excluded.

Seventy-eight patients were considered for patients sampling, among them, 72 patients fulfilled the inclusion criteria and signed the consent form. The intervention group received $1 \mathrm{gram}$ of omega-3 (containing $180 \mathrm{mg}$ EPA and $120 \mathrm{mg} \mathrm{DHA}$ ) twice a day orally and the placebo group received identical soft gelatin capsule with the same shape and packaging containing liquid paraffin). Both omega-3 supplement and identical placebo were produced by Zahraviß Pharmaceutical Company, Tabriz, Iran.

For baseline data gathering, we recruited the patients and asked them to fill all questionnaires at the end of first week. Then, in the second week of follow-up period, we administered the omega-3 or placebo randomly twice daily for 4 weeks. The randomization of samples was carried out based on the blocked randomization method. Information such as the number of treatment groups (2 main intervention groups for example A and placebo for example B), block size (a multiple of the number of groups that were selected in this study) and total patients (the sample size of 60 people) were entered into the internetbased software machines to assigned specific codes to each patients in order to receive omega-3 or placebo soft gelatin capsule. Block randomization was commonly used to balance the number of samples assigned to each of the studied groups when there was sample size limitation. After completing the sample size allocation, the code of each patient was opened and matched with the software output. 
For keeping the study blinded, the data collector, the investigators and the statist were not informed of allocated drug code before data analyses.

\section{Questionnaires:}

At the end of 4-week follow-up, all questionnaires including center for epidemiological studies-depression scale (CES-D) [14], profile of mood states (POMS) [15], Pittsburgh sleep quality index (PSQI) [16] questionnaires were applied again and the results were compared with the baseline.

Center for epidemiological studies-depression scale is a brief, 20-question, self-report scale designed to measure self-reported symptoms associated with depression in six different scales of depressed mood, feelings of guilt and worthlessness, feelings of helplessness and hopelessness, psychomotor retardation, loss of appetite, and sleep disturbance which were experienced in the past week.

The POMS is a clinical psychological rating scale used to assess transient, distinct mood states in 40item questionnaire. Options of this tool are rated from zero (I do not feel that way at all) to 4 (I have that feeling too much).

The last assessed questionnaire was PSQI which has 10 different questions about sleep quality to be asked from the patient in the past month. It is an effective self-administrated instrument includes 4 openended questions and 14 questions to be answered using event-frequency and semantic scales. The tool looks at seven areas: subjective sleep quality, habitual sleep efficiency, sleep disturbances, the use of sleep-promoting medication and daytime dysfunction.

To assess the validity of the Persian version of the questionnaire, it was evaluated by a group of specialists, including two psychologists, one literature expert, two oncologists, and 10 general physicians. Then, a forward-backward translation method was employed for translating the questionnaires, and the same questionnaires were given to 30 matched patients with 2 weeks apart. And finally, the reliability of the questionnaires with an intraclass correlation coefficient (ICC) were assessed. Those patients who were not consumed at least $80 \%$ of total cumulative doses of omega-3 or identical placebo were considered as non-adherent patients and were excluded from the study. In addition, we evaluated every reported adverse drug reaction based on common terminology criteria for adverse events (CTCAE) version 4 during 4-weeks' follow-up $[17,18]$. For the simple review of the proposed method, the graphical abstract was shown in Figure 1.

\section{Statistical Approach:}

\section{Sample size calculation:}

The following equation has been used to determine the number of experimental samples in this research and to investigate the changes of a specific parameter that is used in two different modes [19]. 


$$
\mathrm{N}=\frac{\left(z_{1}+z_{2}\right)^{2}\left[p_{1}\left(1-p_{1}\right)+p_{2}\left(1-p_{2}\right)\right]}{d^{2}}
$$

In this equation, $\mathrm{N}$ indicates the number of patients, is the reliability coefficient (95\%), is the power factor $(80 \%)$, and is the probability of beta power in the first and second groups (50\%), and $d$ is called the study error.

$$
\mathrm{N}=\frac{(1.96+0.84)^{2}[0.5(0.5)+0.5(0.5)]}{(0.35)^{2}} \cong 32
$$

Due to difficulties in reaching optimal sample size, and based on the inclusion criteria and subjects who dropped out in addition to missing data, the authors had to terminate the study by fewer samples, adopting a pilot study design (See flow diagram).

\section{Statistical analysis:}

Per-protocol analysis was considered for comparison the data of those patients who completed the treatment originally allocated. In the data analysis stage, Kolmogorov-Smirnov test was used to evaluate the normality of data distribution in both intervention and placebo groups (patients' demographic and clinical characteristics). Also, in order to evaluate the homogeneity of the distribution parameters in two groups chi-squared were applied. The differences between two groups were assessed by paired t-test, analysis of variance with repeated observations and Fisher's exact test. Paired-sample t-test was applied for analyzing the mean scores of CES-D, POMS, and PSQI questionnaires within a group post and preintervention. In this study, the Statistical Package for Social Sciences (SPSS) version 20 software was used and P-value less than 0.05 was considered significant.

\section{Results}

During 1-year follow-up, 78 patients were included in the study (Figure 2); however, 18 patients were excluded due to lack of cooperation or following reasons: have not drug adherence, disease progression and death.

Thereafter, we divided 60 remained patients into intervention (32 patients) or placebo groups (28 patients) randomly. Clinical and demographic characteristics of patients were shown in Table 1. There were no significant differences in baseline characteristics in term of age, menopausal status, underlying diseases, pathological type of cancer, baseline mean HADS scores and previous treatments regimens between two compared groups. The mean age was $44.5 \pm 10.3$ years in the intervention group and $46.6 \pm 9.9$ years in the placebo group. There were not significantly difference between the two groups in variables $(P>0.42)$. 
Table 1

Baseline clinical and demographic characteristics of patients in intervention and placebo groups $(\mathrm{N}=60)$. Intervention group ( $\mathrm{N}=32)$ Control group $(\mathrm{N}=28)$

\section{Variables}

Pathological type of malignancy

Family history of cance

Underlying diseases

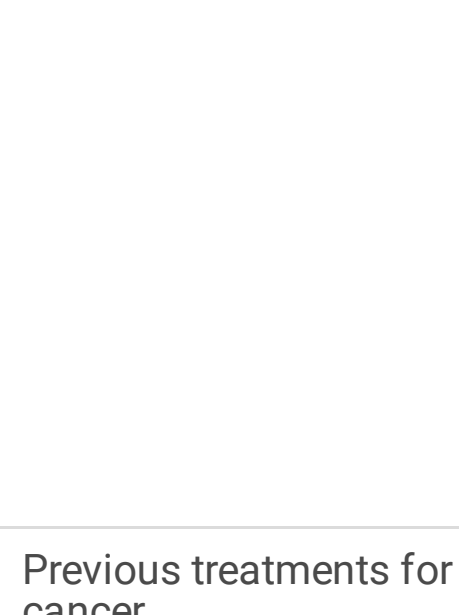

cancer
Age (years)

$44.5 \pm 10.346 .6 \pm 9.9$

Ductal

Lobular

Yes

No

None

Diabetes

Hypertension

Hypothyroidism

Diabetes and Hypertension

Hyperlipidemia

Hyperlipidemia and Diabetes

Chemotherapy

Radiotherapy

Both

Hormone therapy

Menopausal status
Letrozole

Exemestane

Tamoxifen

Letrozole and Goserelin

Tamoxifen and Goserelin

Exemestan and Goserelin

Pre/Perimenopause

Postmenopausal time since diagnosis
$\begin{array}{ll}N(\%) / \text { Mean } & N(\%) / \text { Mean } \\ \pm S D & \pm S D\end{array}$

value

$\begin{array}{ll}29(90.6) & 25(89.3) \\ 3(9.4) & 3(10.7)\end{array}$

13 (40.6)

10 (35.7)

0.70

19 (59.4)

18 (64.3)

20 (62.5)

17 (60.7)

0.28

1 (3.1)

4 (14.3)

6 (18.8)

2 (7.1)

1 (3.1)

1 (3.6)

1 (3.6)

0

$2(7.10$

4 (12.5)

1 (3.6)

9 (28.1)

3 (10.7)

0.17

6 (18.8)

4 (14.3)

17 (53.1)

21 (75)

4 (12.5)

$8(28.3)$

0.10

1 (3.1)

0

1 (3.1)

5 (17.9)

5 (15.6)

3 (10.7)

20 (62.5)

12 (42.9)

1 (3.1)

0

9 (28.1)

10 (35.7)

0.53

23 (71.9)

18 (64.3) 


\begin{tabular}{|l|lll|}
\hline Intervention group ( $\mathbf{N}=\mathbf{3 2})$ Control group $(\mathbf{N}=\mathbf{2 8})$ & \multicolumn{2}{c|}{} & $\begin{array}{l}\mathbf{P}- \\
\text { value }\end{array}$ \\
\hline Variables & $\begin{array}{c}\mathbf{N}(\%) / \text { Mean } \\
\mathbf{\pm S D}\end{array}$ & $\begin{array}{l}\mathbf{N}(\%) / \text { Mean } \\
\mathbf{\pm S D}\end{array}$ & \\
\hline $\begin{array}{l}\text { Baseline mean HADS } \\
\text { scores }\end{array}$ & $17.2 \pm 2.2$ & $16.7 \pm 1.9$ & 0.45 \\
\hline HADS: Hospital anxiety and depression scale, SD: Standard Deviation & & \\
\hline
\end{tabular}

Results of independent samples t-test showed that before the intervention, mean scores of CES-D ( $P=$ 0.97), POMS ( $P=0.88)$, and PSQI $(P=0.71)$ were not significantly different between two groups. But after the intervention, mean scores of CES-D $(P=0.002)$, POMS $(P=0.03)$, and PSQI $(P=0.04)$ were significantly lower in the intervention group than the control group (Table 2 ). 
Table 2

Comparison among scores of center for epidemiological studies-depression scale, profile of mood states,

Pittsburgh sleep quality index at the baseline and after 4-week follow-up between intervention and placebo groups.

\begin{tabular}{|c|c|c|c|c|}
\hline & Variable (Score) & $\begin{array}{l}\text { Before } \\
\text { Intervention } \\
\text { (mean } \pm S D)\end{array}$ & $\begin{array}{l}\text { After } \\
\text { Intervention } \\
\text { (mean } \pm S D)\end{array}$ & $\begin{array}{l}\text { P-value } \\
\text { between before and after } \\
\text { the intervention }\end{array}$ \\
\hline \multirow{10}{*}{$\begin{array}{l}\text { Intervention } \\
\text { Group }\end{array}$} & CES-D & $30.8 \pm 11.9$ & $22.8 \pm 8.8$ & $0.001>$ \\
\hline & POMS & $39.5 \pm 16.9$ & $30.8 \pm 11.1$ & $0.001>$ \\
\hline & PSQI & $5.9 \pm 2.4$ & $4.6 \pm 2.2$ & 0.04 \\
\hline & Subjective Sleep Quality & $1.4 \pm 0.6$ & $0.8 \pm 0.4$ & $0.001>$ \\
\hline & Delay in Falling Asleep & $1.61 \pm 0.8$ & $1.1 \pm 0.7$ & $0.001>$ \\
\hline & Duration of Sleep & $1.2 \pm 0.9$ & $1.1 \pm 0.9$ & 0.22 \\
\hline & $\begin{array}{l}\text { Habitual Sleep } \\
\text { Efficiency }\end{array}$ & $0.3 \pm 0.1$ & $0.31 \pm 0.1$ & 0.99 \\
\hline & Sleep Disturbances & $1.1 \pm 0.5$ & $0.8 \pm 0.3$ & 0.04 \\
\hline & $\begin{array}{l}\text { Use of Sleep-Promoting } \\
\text { Medication }\end{array}$ & 0 & 0 & 1 \\
\hline & Daytime Dysfunction & $0.5 \pm 0.2$ & $0.3 \pm 0.1$ & 0.14 \\
\hline \multirow{10}{*}{$\begin{array}{l}\text { Control } \\
\text { Group }\end{array}$} & CES-D & $30.7 \pm 8.9$ & $29.5 \pm 7.3$ & 0.32 \\
\hline & POMS & $38.9 \pm 15.2$ & $37.6 \pm 13.2$ & 0.22 \\
\hline & PSQI & $6.19 \pm 3.02$ & $6.17 \pm 3.03$ & 0.71 \\
\hline & Subjective Sleep Quality & $1.5 \pm 0.8$ & $1.4 \pm 0.8$ & 0.26 \\
\hline & Delay in Falling Asleep & $1.62 \pm 0.7$ & $1.6 \pm 0.9$ & 0.62 \\
\hline & Duration of Sleep & $1.3 \pm 1.01$ & $1.4 \pm 1.01$ & 0.29 \\
\hline & $\begin{array}{l}\text { Habitual Sleep } \\
\text { Efficiency }\end{array}$ & $0.03 \pm 0.1$ & $0.29 \pm 0.1$ & 0.06 \\
\hline & Sleep Disturbances & $1.4 \pm 0.6$ & $1.3 \pm 0.5$ & 0.28 \\
\hline & $\begin{array}{l}\text { Use of Sleep-Promoting } \\
\text { Medication }\end{array}$ & $0.07 \pm 0.05$ & $0.04 \pm 0.03$ & 0.23 \\
\hline & Daytime Dysfunction & $0.3 \pm 0.1$ & $0.2 \pm 0.08$ & 0.33 \\
\hline
\end{tabular}

CES-D: Center for epidemiological studies-depression scale, POMS: Profile of mood states, PSQI: Pittsburgh sleep quality index, SD: Standard Deviation 
Table 3

Comparison among scores of center for epidemiological studies-depression scale, profile of mood states, Pittsburgh sleep quality index at the baseline and after 4-week follow-up within both intervention and placebo groups.

\begin{tabular}{|c|c|c|c|c|c|c|}
\hline \multirow[t]{2}{*}{ Variables (Scores) } & \multicolumn{2}{|c|}{$\begin{array}{l}\text { Intervention group } \\
\text { (mean } \pm \mathrm{SD} \text { ) }\end{array}$} & \multicolumn{2}{|c|}{$\begin{array}{l}\text { Placebo group } \\
\text { (mean } \pm \text { SD) }\end{array}$} & \multirow{2}{*}{$\begin{array}{l}\text { P-value } \\
\text { Inter } \\
\text { group } \\
\text { before } \\
4 \\
\text { weeks }\end{array}$} & \multirow{2}{*}{$\begin{array}{l}\text { P- } \\
\text { value } \\
\text { Inter } \\
\text { group } \\
\text { after } 4 \\
\text { weeks }\end{array}$} \\
\hline & Baseline & 4 weeks & Baseline & 4 weeks & & \\
\hline CES-D & $30.8 \pm 11.9$ & $\begin{array}{l}22.8 \pm \\
8.8\end{array}$ & $30.7 \pm 8.9$ & $29.5 \pm 7.3$ & 0.97 & 0.002 \\
\hline POMS & $39.5 \pm 16.9$ & $\begin{array}{l}30.8 \pm \\
11.1\end{array}$ & $\begin{array}{l}38.9 \pm \\
15.2\end{array}$ & $37.6 \pm 13.2$ & 0.88 & 0.03 \\
\hline PSQI & $5.9 \pm 2.4$ & $4.6 \pm 2.2$ & $6.19 \pm$ & $6.17 \pm 3.3$ & 0.71 & 0.04 \\
\hline Subjective Sleep Quality & $1.4 \pm 0.6$ & $0.8 \pm 0.4$ & & $1.4 \pm 0.8$ & 0.53 & 0.002 \\
\hline Delay in Falling Asleep & $1.61 \pm 0.8$ & $1.1 \pm 0.7$ & & $1.6 \pm 0.9$ & 0.91 & 0.02 \\
\hline Duration of Sleep & $1.2 \pm 0.9$ & $1.1 \pm 0.9$ & 1101 & $1.4 \pm 1.01$ & 0.76 & 0.26 \\
\hline Habitual Sleep Efficiency & $0.3 \pm 0.1$ & $0.31 \pm 0.1$ & $\cap \cap 3+\cap 1$ & $0.29 \pm 0.1$ & 0.16 & 0.89 \\
\hline Sleep Disturbances & $1.1 \pm 0.5$ & $0.8 \pm 0.3$ & $14+06$ & $1.3 \pm 0.5$ & 0.09 & 0.005 \\
\hline $\begin{array}{l}\text { Use of Sleep-Promoting } \\
\text { Medication }\end{array}$ & 0 & 0 & $0.07 \pm 0.05$ & $0.04 \pm 0.03$ & 0.13 & 0.33 \\
\hline Daytime Dysfunction & $0.5 \pm 0.2$ & $0.3 \pm 0.1$ & $0.3 \pm 0.1$ & $0.2 \pm 0.08$ & 0.44 & 0.53 \\
\hline
\end{tabular}

According to figure 2, based on results from CESD and POMS questionnaires, positive changes (mean and standard deviation) have been demonstrated with respect to the normal histogram distribution.

Results of paired-sample t-test showed that in the intervention group, mean scores of CES-D $(P<0.001)$, POMS $(P<0.001)$, and PSQI $(P=0.003)$ were significantly lower in post-intervention than pre-intervention. However, in the control group, mean scores of CES-D $(P=0.32)$, POMS $(P=0.22)$, and PSQI $(P=0.96)$ were not significantly different pre- and post- intervention (Table 3 ).

Results of independent-samples t-test showed no significant difference in mean scores of none of the subgroup in sleep quality disorder before intervention between two groups $(P>0.05)$. However, after the intervention, mean score of sleep quality disorder in areas of mental quality of sleep $(P=0.002)$, delay in falling asleep $(P=0.02)$, and sleep disorders $(P=0.005)$ were significantly lower in the intervention group 
than the control group, but mean scores of other areas of sleep quality disorder were not significantly different between two groups (Table 3).

Results of paired samples t-test showed that in the intervention group, mean scores of areas of mental quality of sleep $(P<0.001)$, delay in falling asleep $(P<0.001)$, and sleep disorders $(P=0.04)$ were significantly lower post-intervention than pre-intervention. But, mean scores of other areas of sleep quality disorder were not significantly different pre- and post- intervention $(P>0.05)$. In the control group, mean scores of sleep quality disorders were not significantly different pre- and post-intervention (Table $3)$.

According to CTCAE version 4[17], no noteworthy adverse reaction was noted during 4-week administration of $2 \mathrm{~g}$ omega-3 daily either in intervention group or placebo ones.

\section{Discussion}

For the first time in this study, we have demonstrated that administration of omega- 3 for 4 weeks was associated with a significant increase in sleep quality scores, delayed sleep phase, and sleep disturbance, and also a significant increase in mean changes in CES-D and POMS scores compared to the placebo. In an open-label study conducted by Freeman et al. in 2011 [20] the impact of 2 gram daily omega-3 supplementation for 8-week follow-up on the menopausal symptoms including psychological symptoms of depression and hot flashes were assessed, results show that omega-3 supplementation caused decrease in a response rate of Montgomery-Asberg depression rating scale (MADRS) and hot flash related daily Interference scale scores significantly $(P=0.006)$. Furthermore, the positive effects of omega-3 supplementation on psychiatric problems induced by anti-hormone therapy were demonstrated in this study. Furthermore, administration of omega- 3 can be considered for psychiatric problems such as depression, anxiety, mood and sleep disorder. The effective impact of omega-3 on enhancing depression, mood changes and sleep disturbance and quality were confirmed in our study by assessing standard questionnaires. There are several studies addressed the positive effects of omega-3 supplementation on behavioral, cognitive or mood disorders.

For instance, in a study on patients with lung cancer in Japan, it was found that alpha-lipoic acid levels and overall omega-3 intake were inversely related to incidence of depression occurrences [8]. Smith et al., (2017) reported that low-dose omega-3 supplements used to treat depression reduced insomnia, and even improved quality of life of the depressed patients who did not respond well to routine treatments [21]. Results of a cross-sectional study also showed that omega-3 supplementation at moderate doses reduced prevalence of depression in patients with depression [22]. Finally, Orchard et al., (2016) indicated that omega-3 supplementation would reduce behavioral, cognitive, and mood disorders, such as depression in patients with cancer [23].

There are also animal studies that have led to neuroprotective effects and the improved brain and mood function in rats including the study by Larrieu et al., in which administered of omega-3 fatty acids led to 
the brain function improvement, depression and anxiety enhancement, and especially positive effects on sensory receptors perception in animals' model [22].

Finally, in a review study conducted in 2019, considering all the evidence, it was concluded that the effects of omega-3 supplementation in animal studies were sufficiently provocative and this supplement were able to lead a reduction in depression scores in the animal model. Hence, this evidence could be extrapolated to clinical condition and the hopeful results should be evaluated more carefully in the clinical setting [24].

Molecular and biological heterogeneity of breast cancer in humans emphasized the need for administration of a multifunctional method for effective chemotherapy prevention. Estrogen receptors reduce incidence of estrogen-receptor-positive tumors under the influence of anti-estrogen drugs, such as tamoxifen and raloxifene but they were ineffective against growth of hormone-independent cancers. Studies have shown that omega-3 fatty acids suppressed cell growth by inducing apoptosis in MCF-7 cells (breast cancer cell line). Omega- 3 in combination with tamoxifen has been also demonstrated to reduce tamoxifen-induced cell apoptosis [23]. Omega-3 administration had some positive effects on hot flashes and depression, as common complications of menopause. This substance was likely to positively cover the menopausal complications caused by hormone therapies in patients with breast cancer $[25,26]$. In fact, psychomotor changes due to the use of sex hormone-lowering drugs were a long-term complication that reduced quality of life of patients who needed medical intervention in severe cases.

In the present study, mean scores of sub-scales for sleep quality, sleep delay, and sleep disorders as well as mean changes in CES-D and POMS scores were significantly different in the omega 3-treated (intervention) group pre- and post-intervention indicating an increase in quality of life in the field of sleep following administration of omega-3 in patients with breast cancer.

As far as our knowledge served, for the first time, it can be concluded that omega- 3 has the potential to reduce mood disorders as well as to improve sleep quality and duration of sleep in patients with breast cancer. Therefore, this drug can be considered as a supplement to treat mood disorders and sleep quality indicators in these patients. However, due to paucity of data, the exact mechanism of omega-3 supplementation on mood and sleep disorder has not yet understood and should be considered for pharmacological and molecular future studies.

Given small sample size and short duration of the study, further studies are suggested to perform with a larger sample size and longer study follow-up.

\section{Conclusion}

This study showed that omega-3 supplementation can improve mood and sleep disorder in women suffering from breast cancer while they were managing with anti-hormone drugs. Considering the positive effect of omega-3 supplementation on menopausal parameters, quality of life and sexual function score 
in patients suffering from breast cancer, this supplement can be proposed for the treatment of these patients.

\section{Declarations}

\section{Funding:}

This study was part of Pharm D thesis and supported by Isfahan University of Medical Sciences (grant number:399177). All the authors declare no conflict of interest.

\section{Conflicts of interest/Competing interests:}

Nil

Availability of data and material: Not applicable

Code availability:

Not applicable

\section{Authors' contributions:}

A.M and M.H contributed to the literature search, acquisition of data, and writing the original and revised manuscript. Both A.M were responsible for the study design and writing the original and revised manuscript. A.D, E.N, V.M and M.H were responsible for recruiting patients. All authors approved the final paper.

\section{Ethics approval:}

The protocol of study has been approved in Isfahan University of Medical Sciences ethic committee (ID number: IR.MUI.RESEARCH.REC.1399.362) and also trial was submitted in Iranian clinical trial registry center (IRCT20180722040556N6).

\section{Consent to participate:}

Consent form was signed by all participants.

Consent for publication:

Not applicable

\section{References}

1. Bray, F., et al., Global cancer statistics 2018: GLOBOCAN estimates of incidence and mortality worldwide for 36 cancers in 185 countries. CA: a cancer journal for clinicians, 2018. 68(6): p. 394- 
424.

2. Beatson, G., On the treatment of inoperable cases of carcinoma of the mamma: suggestions for a new method of treatment, with illustrative cases. Cancer journal (Villejuif), 1989. 2(10): p. 347-350.

3. Stubbs, C., et al., Do SSRIs and SNRIs reduce the frequency and/or severity of hot flashes in menopausal women. The Journal of the Oklahoma State Medical Association, 2017. 110(5): p. 272.

4. Pandya, K.J., et al., Gabapentin for hot flashes in 420 women with breast cancer: a randomised double-blind placebo-controlled trial. The Lancet, 2005. 366(9488): p. 818-824.

5. Manni, A., et al., Combination of antiestrogens and omega-3 fatty acids for breast cancer prevention. BioMed research international, 2015. 2015.

6. Weylandt, K.H., et al., Omega-3 fatty acids and their lipid mediators: towards an understanding of resolvin and protectin formation. Prostaglandins \& other lipid mediators, 2012. 97(3-4): p. 73-82.

7. Hooper, L., et al., Risks and benefits of omega 3 fats for mortality, cardiovascular disease, and cancer: systematic review. Bmj, 2006. 332(7544): p. 752-760.

8. Manni, A., et al., The Impact of Fish Oil on the Chemopreventive Efficacy of Tamoxifen against Development of N-Methyl-N-Nitrosourea-Induced Rat Mammary Carcinogenesis. Cancer Prevention Research, 2010. 3(3): p. 322-330.

9. Palacios, S., et al., Omega-3 versus isoflavones in the control of vasomotor symptoms in postmenopausal women. Gynecological Endocrinology, 2017. 33(12): p. 951-957.

10. Reed, S.D., et al., Menopausal quality of life: RCT of yoga, exercise, and omega-3 supplements. American journal of obstetrics and gynecology, 2014. 210(3): p. 244. e1-244. e11.

11. Purzand, B., et al., The comparison of the effect of soybean and fish oil on supplementation on menopausal symptoms in postmenopausal women: A randomized, double-blind, placebo-controlled trial. Complementary Therapies in Clinical Practice, 2020. 41: p. 101239.

12. Cohen, L.S., et al., Efficacy of omega-3 treatment for vasomotor symptoms: a randomized controlled trial: omega-3 treatment for vasomotor symptoms. Menopause (New York, NY), 2014. 21(4): p. 347.

13. Montazeri, A., et al., The Hospital Anxiety and Depression Scale (HADS): translation and validation study of the Iranian version. Health and quality of life outcomes, 2003. 1(1): p. 1-5.

14. Radloff, L.S., The CES-D scale: A self-report depression scale for research in the general population. Applied psychological measurement, 1977. 1(3): p. 385-401.

15. McNair, P., M. Lorr, and L. Droppleman, POMS manual. 1981, San Diego: Educational and Industrial Testing Service.

16. Buysse, D.J., et al., The Pittsburgh Sleep Quality Index: a new instrument for psychiatric practice and research. Psychiatry research, 1989. 28(2): p. 193-213.

17. Chen, A.P., et al., Grading dermatologic adverse events of cancer treatments: the Common Terminology Criteria for Adverse Events Version 4.0. Journal of the American Academy of Dermatology, 2012. 67(5): p. 1025-1039. 
18. Group, E.B.C.T.C., Effects of chemotherapy and hormonal therapy for early breast cancer on recurrence and 15-year survival: an overview of the randomised trials. The Lancet, 2005. 365(9472): p. 1687-1717.

19. Sakpal, T., Sample size estimation in clinical trial. Perspectives in clinical research, 2010. 1(2): p. 6767.

20. Freeman, M.P., et al., Omega-3 fatty acids for major depressive disorder associated with the menopausal transition: a preliminary open trial. Menopause (New York, NY), 2011. 18(3): p. 279.

21. Smith, D.J., et al., Adjunctive low-dose docosahexaenoic acid (DHA) for major depression: An openlabel pilot trial. Nutritional neuroscience, 2018. 21(3): p. 224-228.

22. Larrieu, T. and S. Layé, Food for mood: Relevance of nutritional omega-3 fatty acids for depression and anxiety. Frontiers in physiology, 2018. 9: p. 1047.

23. Orchard, T.S., et al., Clearing the fog: a review of the effects of dietary omega-3 fatty acids and added sugars on chemotherapy-induced cognitive deficits. Breast cancer research and treatment, 2017. 161(3): p. 391-398.

24. Freitas, R.D. and M.M. Campos, Protective effects of omega-3 fatty acids in cancer-related complications. Nutrients, 2019. 11(5): p. 945.

25. Lucas, M., et al., Effects of ethyl-eicosapentaenoic acid omega-3 fatty acid supplementation on hot flashes and quality of life among middle-aged women: a double-blind, placebo-controlled, randomized clinical trial. Menopause, 2009. 16(2): p. 357-366.

26. Chalon, S., Omega-3 fatty acids and monoamine neurotransmission. Prostaglandins, Leukotrienes and Essential Fatty Acids, 2006. 75(4-5): p. 259-269.

\section{Figures}


Ilormone positive breast cancer patients' recruitment

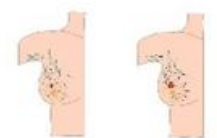

$\frac{2}{4}$
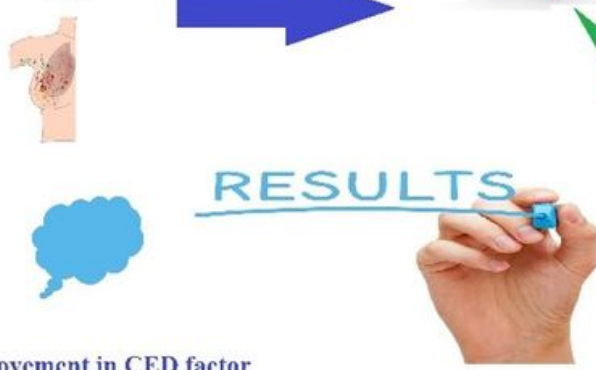

1. Improvement in CED factor

Block randomization into Omega-3 or Identical Placebo

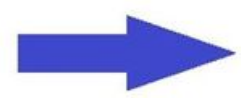

2. Improvement in Profile of Mood State (POMS) Factor

3. Improvement in Subjective Slcep Quality Factor

4. Improvement in Delay Falling asleep Factor

5. Improvement in Sleep Disturbances Factor
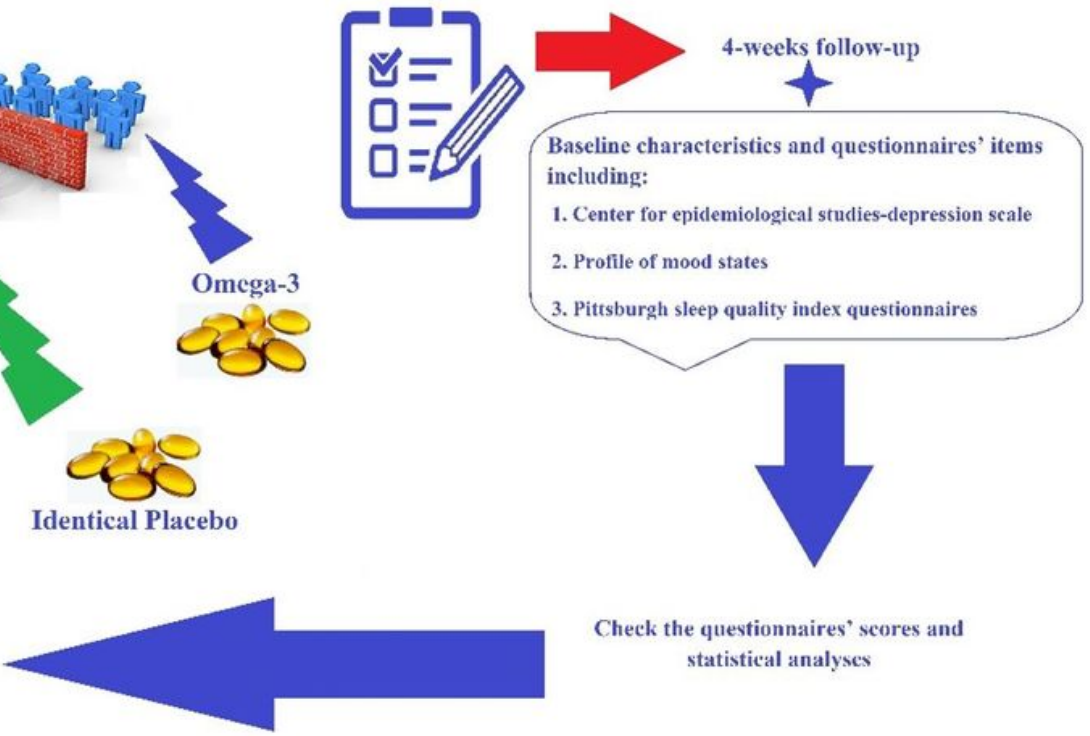

Check the questionnaires' scores and statistical analyses

\section{Figure 1}

Steps of the proposed method 


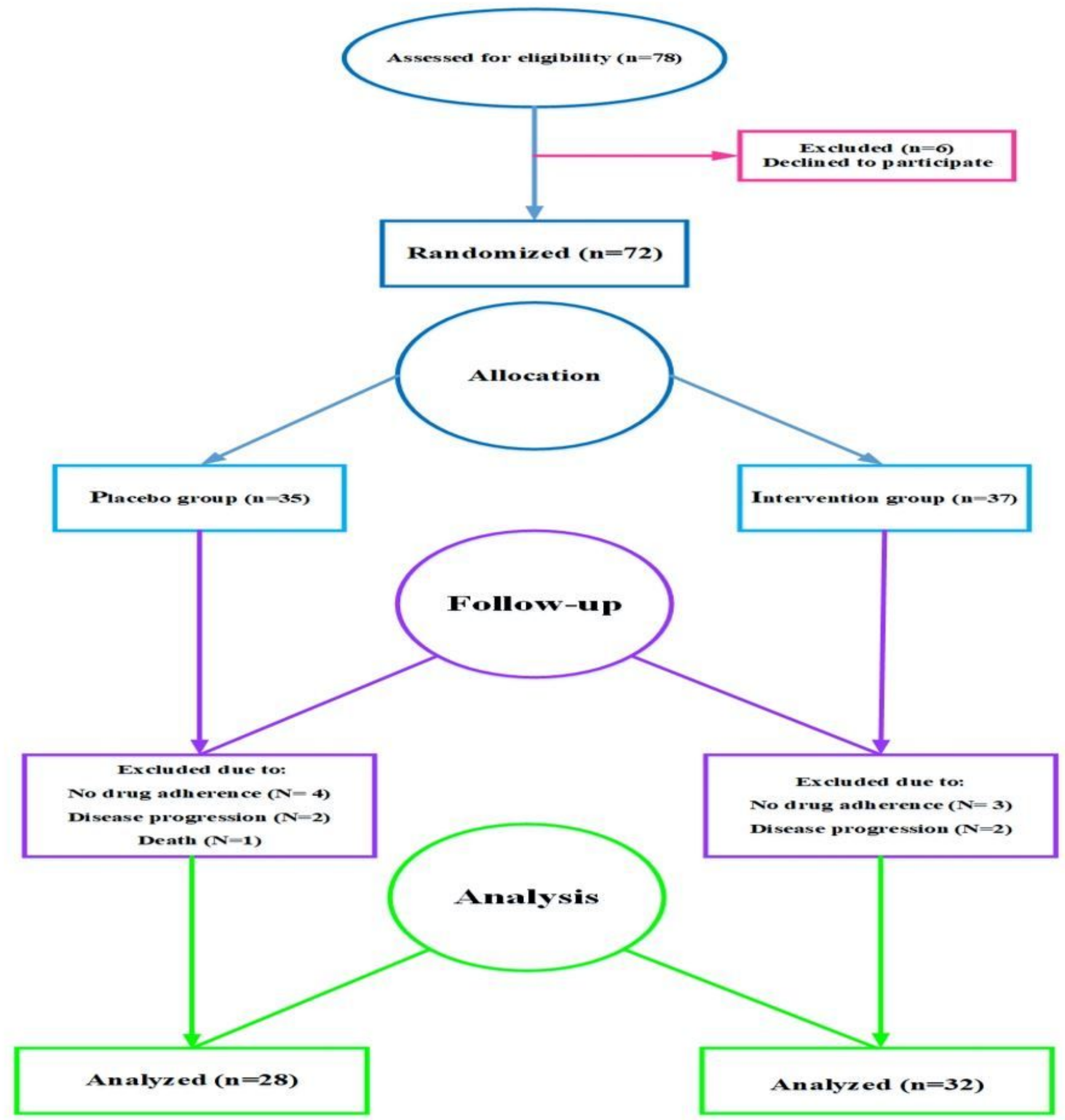

Figure 2

Consolidated standards of reporting trials (CONSORT) flowchart of the study. 


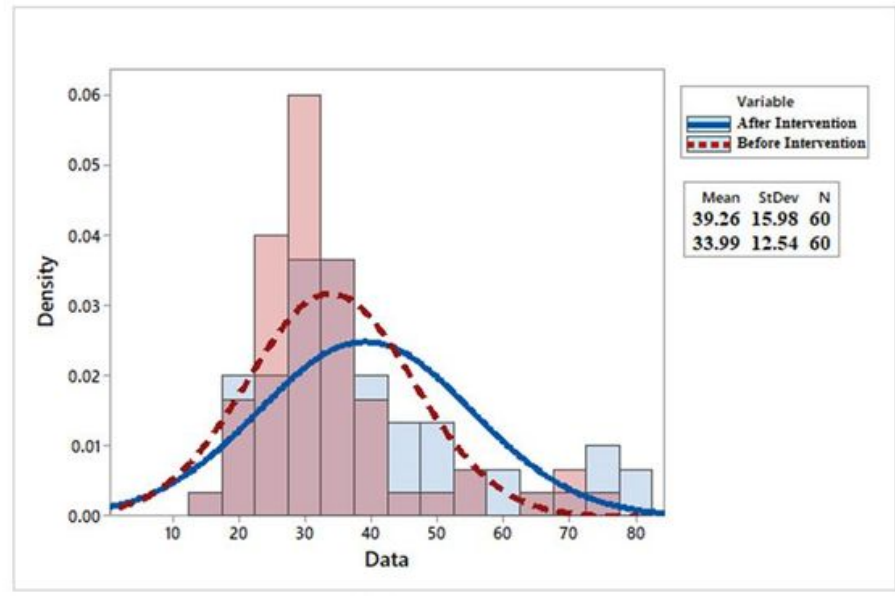

(a)

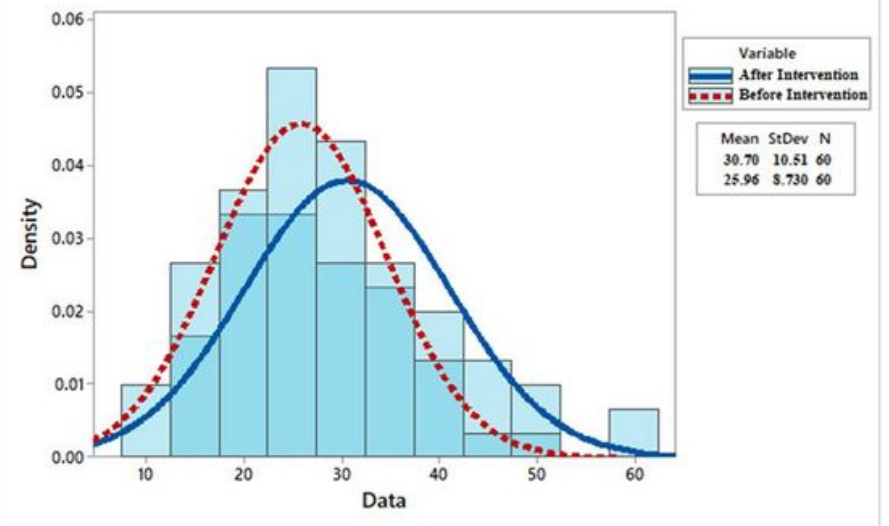

(b)

\section{Figure 3}

Comparison the mean score of center for profile of mood states (a) and center for epidemiological studies-depression (b) scales before and after the omega-3 supplementation in intervention group 